\title{
L'HYGIÈNE DU LAIT AU CANADA
}

par

\author{
E. U. ALLARD \\ Chef inspecteur du lait à Québec.
}

La ville de Québec a été une des premières villes du Dominion. du Canada, ainsi que du continent Nord Américain, à s'occuper, vers 1890 , de la réglementation et du contrôle du lait consommé dans ses limites. Nous voyons dans les archives de la ville, aux délibérations du Comité de Santé, que dans les années de 1891 et 1892 , il est question de la surveillance de la qualité du lait vendu aux eitoyens de la ville.

Pour ce qui est de Montréal, nous lisons dans le "Canadian Public Health Journal » une conférence faite par le $D^{r}$ A. J. G. HooD, M. V., surintendant de la division des aliments du Département de Santé de la ville de Montréal, ce qui suit :

"En 1881, la Société Médicale de Montréal discuta la nécessité de nommer un vétérinaire qui serait chargé de l'inspection du lait, mais ce n'est qu'en 1889 que le premier inspecteur du lait fut nommé. De cette date à 1900 , il n'apparaît pas que l'on ait fait beaucoup de progrès, mais la nomination, en 1900, de deux nouveaux vétérinaires pour l'inspection du lait a apporté un chanment appréciable à la qualité du lait."

$\mathrm{Si}$, à cette époque, nous trouvons, à l'ordre du jour, dans plusieurs grandes villes, cette question du contrôle du lait, e'est dû aux découvertes scientifiques d'alors, découvertes que nous devons aux travaux des Pasteur, des Koch, des Babcock et autres. Elles. ont permis l'organisation de laboratoires capables de nous guider par les différentes analyses et épreuves nécessaires à toute inspection bien contrôlée.

A Québec, le premier règlement concernant le lait date du 2 mars 1893, et la nomination du premier inspecteur de lait du 10 mai 1894.

Le 12 avril 1907 , le premier règlement, rendant obligatoire la tuberculinisation de tous les animaux laitiers fournissant du lait à la ville, était présenté au Conseil de ville et adopté le 26 avril de la même année.

Pendant les vingt premières années, c'est-à-dire de 1893 à 1913 , l'inspection du lait ne semble pas avoir fait de grands progrès, car à part quelques échantillons de lait prélevés irrégulièrement et qui ne servaient qu'à la recherche du pourcentage des matières grasses et des solides totaux du lait, l'on ne trouve rien dans les registres sur l'analyse bactériologique ni sur l'épreuve au sédimentateur 
et à l'acidimètre. Il n'est question, non plus, d'aucun système régulier d'inspection des troupeaux et des fermes pas plus que d'un rapport annuel sur la question du lait.

En 1913, M. le Dr C. O. Guimont, directeur du Laboratoire municipal, signalait aux autorités du temps, le manque de contrôle et de surveillance et l'état lamentable dans lequel étaient la production et la manipulation du lait vendu aux consommateurs de la ville. A la suite d'inspections et d'analyses, il démontra la nécessité d'une règlementation et obtint que de nouveaux inspecteurs soient nommés (je fus un des inspecteurs nommés par le Dr GurMont, et j'entrai à son service le 12 juillet 1914).

M. le $\mathrm{D}^{\mathrm{r}}$ Guimont dirigea le Service de l'Inspection du Lait de 1913 à 1916. Pendant ces trois années, il organisa un système de prélèvement d'échantillons pour l'analyse chimique et bactériologique et un système de fiches pour l'inspection des troupeaux et des fermes. Il rédigea aussi un nouveau règlement concernant le lait qu'il fit adopter par le Conseil de ville, le 30 avril 1915.

Au mois de septembre 1916, j'étais chargé par le $\mathrm{D}^{\mathrm{r}} \mathrm{C}$. R. PAquin, Médecin municipal de la ville, de l'organisation du Service de l'Inspection du lait et des fermes, que nous avons présentement.

Après un voyage à Montréal et aux Etats-Unis à l'automne de 1916, j'entrepris l'amélioration de nos fermes de production de lait, de nos laiteries de pasteurisation, comme aussi de la manipulation et de la vente du lait aux consommateurs, dans le sens préconisé pour le contrôle et l'inspection, dans les autres villes canadiennes et américaines.

Aiu début, ce qui laissait le plus à désirer, c'était la production d'un lait malpropre dans la grande majorité des fermes et l'absence de tout soin à donner au lait après la traite, en vue de sa conservation.

Pendant la stabulation de l'hiver, c'était la coutume, en général, de garder les vaches sans soin, sous le rapport de la toilette ; c'était l'exception de rencontrer le contraire.

L'intérieur des étables n'était jamais blanchi à la chaux, comme la chose se fait maintenant tous les automnes.

L'éclairage, la ventilation, le drainage et les pavés des étables n'avaient jamais été pris en considération.

L'on gardait en quantité dans les étables avec les vaches, des porcs et des volailles; ce qui a toujours un mauvais effet sur les conditions sanitaires d'une vacherie.

L'eau pour le service général dans plusieurs fermes était de qualité douteuse et nous avons eu, dans le passé, des épidémies de typhoïde causées par des approvisionnements d'eau souillée dans les fermes. 
Les ustensiles à lait tels que bidons, chaudières et couloirs. laissaient à désirer sous le rapport des conditions sanitaires.

On utilisait pour le lait tout ce qui pouvait servir d'ustensiles, tels que seaux de bois ou de pulpe, chaudières de tôle galvanisée et autres métaux oxydables et de lavage très difficile.

Mais ce qui était horrible, c'était l'usage que l'on faisait des ustensiles à lait, les chaudières à lait servant en général à tous les besoins de la ferme et souvent aux besognes les plus dégoûtantes.

Quant aux bidons, qu'il nous suffise de dire que nous avons dî lutter pendant quelques années avant de faire cesser une coutume qui consistait pour plusieurs laitiers à rapporter chez eux, au retour de leur vente de lait, des vidanges de cuisine ramassées dans les cours des hôtels, restaurants, etc,, et cela dans les bidons qui servaient à la vente régulière de leur lait; aujourd'hui il est. presque incroyable que cet état de chose ait existé.

Quant au soin à donner au lait, aucune laiterie n'existait chez les producteurs ou chez les laitiers et l'on n'avait pas songé à la. glace pour le refroidissement du lait. Les endroits ordinaires où l'on gardait le lait étaient la cave, la cuisine de la maison, un hangar à débarras, une remise ou un passage quelconque, d'autres n'avaient rien pu trouver de mieux que de garder le lait dans l'étable même, et enfin quelques-uns ne craignaient pas de garder leur lait dans. la porcherie; tous ces endroits étaient condamnables commeentrepôts pour le lait, ne fut-ce qu'à cause des milliers de mouches. qui habitaient ces lieux.

Le lait se livrait sans refroidissement et sans protection contre le soleil, la boue, la poussière et dans les bidons dont nous avons déjà parlé.

Les établissements de pasteurisation qui fournissaient du lait. pasteurisé à la ville, présentaient, sous plusieurs rapports, les mêmes conditions que l'on trouvait chez les laitiers et les producteurs delait du temps.

La science de la pasteurisation n'était pas ce qu'elle est maintenant, les premiers appareils n'avaient pas atteint le degré de perfectionnement que l'on trouve dans ceux d'aujourd'hui et plusieurs des principaux instruments de précision indispensables au contrôle scientifique des différentes opérations de la pasteurisation n'existaient pas. Ajoutons à cela le manque d'organisation d'une industrie naissante, la rareté d'un personnel compétent, et nous aurons une idée du problème qu'il fallait résoudre.

Si la pasteurisation du lait n'a pas été un fiasco complet avec un pareil début, c'est qu'elle était une science que rien ne pouvait. faire mourir. 
Et l'on sait comment cette science de l'hygiène du lait a été attaquée. Un intérêt mesquin, l'ignorance et souvent la mauvaise foi lui ont fait une lutte sans merci.

$\mathrm{Au}$ fur et à mesure que s'organisait le contrôle de l'inspection du lait, des fermes, des établissements de pasteurisation, de la manipulation et de la vente du lait sous le nouveau service de 1916 , le besoin d'une nouvelle règlementation se faisait de plus en plus sentir. Le 9 avril 1920, le Conseil de ville de Québee passait un nouveau règlement concernant le lait. Ce règlement $n^{\circ} 5-G$, qui est encore en vigueur aujourd'hui, contenait 236 articles; et depuis cette date une douzaine de nouveaux articles ont été ajoutés à ce règlement par différents amendements.

Pour obtenir ces améliorations, il a fallu que les inspecteurs fussent en même temps des " instructeurs ». Le travail des inspecteurs ne s'est pas borné simplement à des constatations et à des rapports, mais il fallait que l'inspecteur fût capable de démontrer aux producteurs et aux laitiers, ce qui faisait défaut dans leur organisation et leur routine en rapport avec les règlements d'hygiène. De plus, ils devaient être en état de les conseiller et de les guider quant aux améliorations à faire. Tel fut le cas pour la confection et le soin des ustensiles, pour l'éclairage, la ventilation, le blanchissage et les soins de propreté aux étables et aux vaches, pour les soins à donner au lait, à la traite, au refroidissement, à la livraison, ou pour toute autre manipulation, la construction de glacières, l'amélioration de la qualité de l'eau dans les fermes. Ces travaux d'instruction se sont aussi appliqués à l'amélioration de nos établissements de pasteurisation.

Les améliorations dans les fermes ont progressé dans l'ordre suivant :

Amélioration des ustensiles à lait : tous les mauvais ustensiles ont été, premièrement, mis de côté ou confisqués et nous avons exigé qu'à l'avenir, les ustensiles à lait seraient faits de métaux spéciaux (cuivre, acier ou tôle étamée et autres métaux non oxydables) et confectionnés de façon à être facile à laver et à stériliser. Défense fut faite aux producteurs et laitiers de faire servir les ustensiles à lait à autre chose qu'au service du lait.

Certaines années, nous avons dû confisquer de grandes quantités d'ustensiles à lait en mauvaise condition, et ces confiscations ont servi d'exemples et nous ont permis d'obtenir des résultats qui n'auraient pas été atteints autrement.

La propreté des vaches pendant la stabulation de l'hiver a donné occasion au début à de nombreuses discussions, plusieurs producteurs soutenant qu'il était impossible d'avoir des vaches toujours propres 
en hiver. Là encore, il a fallu faire des exemples, soit en annulant les permis ou licences, soit en confisquant le lait. Parfois la Cour du Recorder dut trancher de la question.

L'amélioration de l'éclairage, de la ventilation, le blanchissage de l'intérieur des étables, la propreté de ces dernières, l'isolement des vaches des autres animaux sont venus en troisième lieu.

La construction de laiteries-glacières, comme endroits spéciaux pour manipuler et garder le lait, était quelque chose de nouveau que la plupart des producteurs et laitiers ont cherché à éliminer ou retarder autant que possible.

Nous avons exigé que chaque producteur de lait ou chaque laitier ait une laiterie-glacière où serait manipulé et gardé le lait et c'est surtout important au cas de maladies contagieuses dans les familles des producteurs et laitiers.

Ces laiteries ne sont acceptées que lorsqu'elles sont construites isolées des étables, porcheries, etc., et de tout lieu de contamination et sans communication avec une maison habitée. Et elles doivent être construites de façon à pouvoir servir au soin du lait tous les jours de l'année, sans égard aux saisons.

Des plans ont été fournis par le Service de l'Inspection du lait pour la construction de la glacière, de la laiterie et des bassins de refroidissement pour le lait. En plus de ces plans, les inspecteurs vont sur place examiner les lieux et donner les instructions nécessaires pour que le tout soit conforme au règlement.

La construction de ces laiteries-glacières nous a permis d'exiger le refroidissement du lait à une basse température après la traite et sa conservation à moins de $50^{\circ}$ F. (1), chez le producteur, pendant le transport, et à la livraison au consommateur.

Nous avons fait des centaines de démonstrations dans les fermes, en présence des producteurs de lait, pour leur enseigner comment produire un lait propre et comment traiter le lait pour lui garder ses bonnes qualités jusque chez le consommateur.

Nous avons fait aussi un travail spécial pour améliorer la qualité de l'eau en usage dans les fermes. Nous avons exigé que les puits ou sources d'approvisionnement soient protégés contre les eaux souillées et dans le cas où l'eau provenait d'aqueduc privé ou public, nous en avons fait l'inspection avec les officiers du Service Provincial d'Hygiène. Dans plusieurs endroits où les améliorations suggérées conformément à la loi d'hygiène provinciale n'ont pas été acceptées, il a été de notre devoir, après un délai raisonnable, de révoquer la licence ou le permis de ceux qui ne s'étaient pas rendus à nos demandes.

(1) $10^{\circ}$ Centigrades. 
Il nous a fallu, dans certains cas, annuler les permis de tout un groupe de cultivateurs qui s'approvisionnaient au même endroit où l'eau était de qualité douteuse.

Pour l'amélioration des établissements de pasteurisation - du lait, nous avons procédé de la même façon que pour les fermes de producteurs et pour les laitiers. Nous avons exigé que les machines, appareils et instruments qui servaient au lait fussent de bonne condition et de capacité suffisante pour répondre aux exigences du règlement. Conformément à ces mêmes exigences, des améliorations aux établissements déjà existants, et même des constructions nouvelles ont été imposées. Nous avons travaillé à instruire le personnel au moyen de publications, de conférences et de démonstrations sur le devoir et les responsabilités de chacun.

Nous avons, de plus, obtenu de l'honorable Ministre de l'Agriculture, M. Gopbout, que des cours spéciaux soient donnés à l'Ecole d'Industrie laitière provinciale à Saint-Hyacinthe, pour former des techniciens compétents capables de prendre charge des établissements de pasteurisation.

Et dès le premier de ces cours donné à l'automne de 1932, quatre de nos établissements de pasteurisation envoyaient à leurs frais, pendant plus de deux mois, les contremaîtres de la pasteurisation, pour les mieux qualifier.

Tout ceci a permis à nos établissements de pasteurisation de. rencontrer sans difficulté les exigences de la nouvelle loi provinciale (Loi David de 1928) sur les établissements de pasteurisation de lait dans la province, et depuis, chaque fois qu'ils ont été visités par des officiers provinciaux chargés de l'application de cette loi, ils ont toujours été classés de premier ordre.

Toute cette organisation, tout ce travail auraient été relativement faciles et agréables, si nous n'avions pas eu à déplorer trop souvent des critiques et une opposition venant de personnes qui, par leurs fonctions et leurs responsabilités, nous devaient plutôt leur appui et leur encouragement.

D'autres personnes, peut-être de bonne foi celles-là, critiquaient nos exigences sous prétexte que les améliorations demandées au nom de l'hygiène du lait, auraient pour résultat d'en faire monter le prix au détriment du consommateur pauvre; on allait jusqu' parler de disette de lait pour la ville si nos exigences étaient maintenues.

Pourtant ce fut de 1930 à 1933, alors que la plupart des améliorations que nous avions demandées au commerce du lait étaient en grande partie terminées, que l'on vit à Québec la plus grande abondance de lait et les prix les plus bas depuis vingt ans. Nous étions donc bien loin de la disette et des hauts prix prédits à ce sujet. 
Ce qui montre bien que notre travail et nos exigences, loin de nuire au commerce du lait, lui ont été plutôt d'un grand secours, c'est le fait que la consommation du lait "per capita" a doublé depuis 1915.

Les intéressés à Québec sont les premiers à reconnaître ces choses ; tous savent qu'un lait produit dans les conditions d'avant 1915, ne trouverait aujourd'hui que de très rares clients à Québec.

\section{Commerce.}

Aujourd'hui le commerce du lait de la ville de Québec est divisé entre les marchands (laitiers) dans les proportions suivantes: pour le lait pasteurisé : $54 \%$, pour le lait eru : $46 \%$. Mais si nous tenons compte du commerce du lait, de la crème et de la crème glacée, la proportion s'établit à $60 \%$ en faveur des marchands de produits pasteurisés.

Le champ de production qui alimente présentement le commerce du lait de la ville de Québec se compose de troupeaux de 609 fermes, fournissant le lait qui vient de la campagne, et 39 troupeaux gardés par les laitiers résidant dans les limites de la Cité ; ce qui fait un total de 648 troupeaux. Les 609 fermes de la campagne sont situées dans les comtés suivants : pour la rive nord, les comtés de Portneuf, Québec et Montmorency; pour la rive sud, les comtés de Richmond, Arthabaska, Mégantic, Lévis, Bellechasse et Montmagny.

Les troupeaux sont partagés comme suit : 314 troupeaux fournissant le lait vendu cru aux consommateurs et 334 troupeaux fournissant le lait aux établissements de pasteurisation.

Tout le lait cru est vendu en bidons, à l'exception du lait certifié et aussi celui de deux laitiers qui le vendent en bouteilles, ce qui fait moins de $1 \%$ du total du lait cru, vendu en bouteilles.

Cette vente du lait eru en bidons se fait dans des bidons d'une capacité de $2,4,5$ et 8 gallons. Le lait est transvasé au fur et à mesure pendant la livraison pour chaque client.

Pour le lait pasteurisé, la vente se fait en bouteilles chez tous les clients, à l'exception du lait vendu pour être consommé dans les grands hôtels et dans quelques restaurants, pensions, etc. Cette vente de lait pasteurisé en bidons représente environ $1 \%$ de la vente totale.

Tout le lait vendu en détail par un magasin quelconque (épiceries, restaurants, etc.) pour le service d'une clientèle du dehors doit être vendu en bouteilles seulement. Le nombre de ces endroits de détail est de 950 et une inspection y est faite régulièrement. Un permis est accordé par l'inspecteur, si les conditions sont trouvées bonnes, sinon, des améliorations doivent être faites.

Ces magasins doivent être pourvus de glacières propres pour garder le lait à une basse température telle qu'exigée parlerèglement. 


\section{Inspection.}

Le présent système d'inspection répartit le travail des inspecteurs comme suit :

Deux vétérinaires pour l'inspection des troupeaux et des fermes. Deux inspecteurs pour les établissements de pasteurisation et l'arrivée du lait de la campagne.

Deux inspecteurs pour l'inspection du lait au moment de la livraison par les rues. Ceci comprend l'inspection du lait livré par les 400 voitures qui font la livraison du lait tous les matins.

Un inspecteur pour le détail du lait dans les épiceries et autres magasins, les hôtels, restaurants, etc., et la vente de la crème sur les marchés publics par les cultivateurs.

Le nombre d'inspections faites et d'échantillons de lait et de crème prélevés chaque année par les inspecteurs se chiffre à plus de de 30.000 .

Lorsque nous analysons cette somme de travail pour en tirer une conclusion pratique, nous constatons que pour donner aux consommateurs de la ville toute la sécurité à laquelle ils ont droit, il y aurait lieu de faire des changements importants dans certaines parties de notre système d'inspection. Ces changements regarderaient. principalement l'inspection du lait cru vendu en bidons, comme on le verra plus loin.

L'inspection des fermes est organisée pour donner des résultats sur lesquels nous pouvons compter. En effet, aujourd'hui, chacune de nos 648 fermes de production est organisée conformément au règlement $\mathrm{du}$ lait, et toute nouvelle ferme doit obtenir un certificat de l'inspecteur des fermes avant qu'un permis de fournir du lait. à un marchand (laitier) de Québec lui soit accordé.

L'inspection des fermes est faite régulièrement et une attention particulière est apportée pour s'assurer de l'entretien et des bonnes conditions qui doivent y exister.

Le service de l'inspection du lait aux établissements de pasteurisation fonctionne de façon à donner des résultats et son efficacité nous offre des garanties sur la protection que doivent en attendre les consommateurs de la ville.

Pour qu'un service d'inspection de lait atteigne le but pour lequel il est organisé et doive être maintenu, il faut que ces résultats ne soient pas simplement de constater que le lait est de bonne ou mauvaise qualité, mais principalement d'écarter de la consommation les laits suspects et dangereux. Sur ce point, les milliers de gallons de lait qui, chaque année, sont confisqués et retournés aux cultivateurs après avoir été dénaturés pour en empêcher la vente, montrent bien que le contrôle de l'inspection du lait à son arrivée aux établissements de pasteurisation atteint son but. Les laits ainsi refusés sont 
les laits sales et visqueux, les laits de goût et d'odeurs anormales, les laits acides ou en fermentation, les laits trop pauvres et les laits mal refroidis ou non refroidis.

Toute cette inspection et ce classement ne sont possibles que si l'inspecteur dispose du temps et des facilités pour accomplir ce travail,

Aux établissements de pasteurisation, l'inspecteur dispose du temps et des facilités nécessaires à son inspection et au classement du lait, tout comme à l'inspection et à la surveillance de la pasteurisation du lait et des autres opérations.

Ce contrôle se fait régulièrement dans chaque établissement toutes les semaines, et des échantillons du lait pasteurisé sont prélevés aux établissements de pasteurisation et dans les voitures au moment de la livraison, pour analyses bactériologiques et chimiques.

Notre présent système d'inspection des fermes, des établissements de pasteurisation et de la vente du lait au détail dans les magasins, nous donne un contrôle qui assure aux consommateurs de la ville une protection réelle et conforme aux lois et règlements d'hygiène.

Mais je regrette d'avoir à déclarer que cette protection n'est pas aussi efficace quant à ce qui regarde le lait vendu cru en bidons, aux consommateurs de la ville.

Le présent système qui consiste à prélever chaque matin des échantillons de lait des bidons des laitiers dans les rues de la ville, est un vieux système qui n'a pas évolué depuis son début, il y a quarante ans. Ce mode d'inspection était défectueux au commencement et il l'est encore davantage.

Les avantages que les inspecteurs du lait trouvent dans les établissements de pasteurisation pour faire un travail efficace d'inspection et de classement n'existent pas lorsque l'inspecteur doit faire son travail dans les rues. L'on comprend facilement qu'une voiture de laitier chargée de bidons de lait qu'un inspecteur arrête pour prélever des échantillons pendant la livraison dans une rue où le trafic est dense, ne peut pas fournir les facilités raisonnables qu'exige l'inspection bien faite du lait.

Ce système ne permet pas à l'inspecteur de faire autre chose que de prélever des échantillons de lait de chaque voiture qu'il rencontre et d'apporter ces échantillons au laboratoire où sont faites les épreuves qui établissent le classement des différents laits. Mais à ce moment il lui est impossible d'empêcher la vente des laits défectueux, car les constatations qu'il faut faire pour établir la qualité du lait sont faites longtemps après que ces laits ont été livrés aux consommateurs, et ces derniers étant inconnus, sont dans l'impossiblité d'être avertis de la qualité du lait qui leur a été fourni. 
Quant au laitier dont le lait a été trouvé défectueux, nous lui adressons un avis de la mauvaise qualité de son lait ou une poursuite est prise contre lui, suivant ce qui semble, dans les circonstances, indiquer sa responsabilité.

Mais de cette façon, le consommateur n'est pas protégé, peu importe que le laitier soit avisé ou poursuivi.

Il faudrait donner aux inspecteurs chargés de l'inspection du lait vendu cru, les mêmes facilités qu'ont les inspecteurs des établissements de pasteurisation pour classer immédiatement et écarter de la consommation les laits de qualité douteuse, telle que la chose se fait présentement pour le lait qui arrive auxétablissements de pasteurisation.

Avec le présent système d'inspection du lait vendu cru aux consommateurs, deux inspecteurs peuvent difficilement atteindre régulièrement plus de dix à douze laitiers par matin et souvent beaucoup moins, cependant qu'avec le système en pratique dans les établissements de pasteurisation, deux inspecteurs peuvent inspecter en moyenne le lait d'au-delà de 100 laitiers par matin et confisquer les laits douteux ou anormaux, etc.

Nous avons confisqué en 1935 des milliers de gallons de lait cru arrivant aux établissements de pasteurisation, tandis que dans le même temps, nous n'avons confisqué que quelques gallons de lait vendu cru aux consommateurs. Ceci ne veut pas dire que la qualité du lait vendu cru en bidons est meilleure que la qualité du lait eru qui arrive, aux établissements de pasteurisation, mais démontre plutôt que les deux systèmes d'inspection ne donnent pas les mêmes résultats, la qualité du lait étant la même.

(A suivre.)

\section{BIBLIOGRAPHIE ANALYTIQUE}

\section{LES LIVRES}

Perspectives in Biochemistry (Vues sur la Biochimie), 31 essais. Hommage à Sir Frederick Gowland Hopkins, des membres anciens et actuels de son laboratoire, présenté par Joseph Needham et David E. Green. Cambridge. At the University Press, 1937. 1 livre relié toile, de $361 \mathrm{p}$. Prix : $15 \mathrm{sh}$. net.

Cet ouvrage a été dédié à Sir F. G. Hopkins, à l'occasion de ses 75 ans, par ses élèves anciens et actuels. Cet hommage au doyen de la biochimie anglaise a pris la forme d'une série de 31 essais qui traitent de la science de la vie dans ses rapports avec la physiologie, la zoologie, l'embryologie, la médecine, la bactériologie et la nutrition. Les rédacteurs de ces essais n'ont pas seulement relaté les progrès les plus récents réalisés dans les différentes 